\title{
Extract of Corallodiscus flabellata attenuates renal fibrosis in SAMP8 mice via the Wnt/ $\beta$-catenin/RAS signaling pathway
}

Bing Cao ${ }^{1,2}$, Mengnan Zeng ${ }^{1,2}$, Yanpo $\mathrm{Si}^{1,2}$, Beibei Zhang ${ }^{1,2}$, Yangyang Wang ${ }^{1,2}$, Ruiqi $\mathrm{Xu}^{1,2}$, Yanjie Huang ${ }^{1,2}$, Weisheng Feng ${ }^{1,2}$ and Xiaoke Zheng ${ }^{1,2,3^{*}}$

\begin{abstract}
Background: Fibrosis is one of the most common pathological features of the aging process of the kidney, and fibrosis in aging kidneys also aggravates the process of chronic kidney disease (CKD). Corallodiscus flabellata B. L. Burtt (C. flabellata, CF) is a commonly used botanical drug in Chinese folklore. However, few studies have reported its pharmacological effects. This study aimed to explore the effect of CF ethanol extract on renal fibrosis in SAMP8 mice and identify potentially active compounds.
\end{abstract}

Methods: Senescence-accelerated mouse-prone 8 (SAMP8) were used as animal models, and different doses of CF were given by gavage for one month. To observe the degree of renal aging in mice using $\beta$-galactosidase staining. Masson staining and the expression levels of Col-I, a-SMA, and FN were used to evaluate the renal fibrosis in mice. The protein expression levels of Nrf2 pathway and Wnt/ $\beta$-catenin/RAS pathway in the kidney were measured. And $\beta$-galactosidase ( $\beta$-gal) induced NRK-52E cells as an in vitro model to screen the active components of CF.

Results: The CF ethanol extract significantly inhibited the activity of renal $\beta$-galactosidase and the expression levels of Col-I, a-SMA, and FN in SAMP8 mice, and improved Masson staining in SAMP8 mice. CF remarkably reduced urinary protein, creatinine, urea nitrogen and serum levels of TNF- $\alpha$ and IL-1 $\beta$ in SAMP8 mice, and significantly increased the levels of SOD and GSH-Px. Moreover, CF activated the Nrf2 pathway and blocked the Wnt/ $\beta$-catenin/RAS pathway in the kidneys of mice. Besides, 3,4-dihydroxyphenylethanol (SDC-0-14, 16) and (3,4-dihydroxyphenylethanol-8-O-[4-Otrans-caffeoyl- $\beta$-D-apiofuranosyl-( $1 \rightarrow 3)-\beta$-D-glucopyranosyl $(1 \rightarrow 6)]-\beta$-D-glucopyranoside (SDC-1-8) were isolated from CF, which reduced the senescence of NRK-52E cells, and maybe the active ingredients of CF playing the antiaging role.

Conclusions: Our experiments illuminated that CF ethanol extract may ameliorate renal fibrosis in SAMP8 mice via the Wnt/B-catenin/RAS pathway. And SDC-0-14,16 and SDC-1-8 may be the material basis for CF to exert anti-renal senescence-related effects.

Keywords: Aging, Corallodiscus flabellata, Kidney, Renal fibrosis, SAMP8, Senescence, Wnt/ $\beta$-catenin/RAS

*Correspondence: zhengxk.2006@163.com

${ }^{3}$ School of Pharmacy, Henan University of Chinese Medicine, 156 Jinshui East Road, 450046 Zhengzhou, China

Full list of author information is available at the end of the article

\section{Introduction}

The global prevalence of chronic kidney disease (CKD) is increasing with the aging of the population and poses a significant burden to society [1-3]. The most common pathological manifestation of CKD is some form of renal fibrosis [4]. Likewise, renal fibrosis is also one of the signs 
of kidney aging [2]. Studies have shown that oxidative stress, inflammation, Wnt/ $\beta$-catenin, renin angiotensin aldosterone system (RAS) and rapamycin (m-TOR) signaling are all related to CKD induced by senescence [5]. Renal fibrosis in aging kidneys is closely related to the activation of $\mathrm{Wnt} / \beta$-catenin and RAS signaling pathway [6]. Upon activation of Wnts, $\beta$-catenin in the cytoplasm is translocated to the nucleus, where it binds to the lymphoid enhancer-binding factor (LEF)/the T-cell factor (TCF) transcription factor family and initiates the transcription of downstream target genes. Interestingly, the promoter region of the RAS gene also contains LEF/ TCF binding sites, which allows $\beta$-catenin to promote the binding of LEF-1 to these sites [7]. Therefore, targeting the Wnt/ $\beta$-catenin/RAS signaling pathway could be a potential therapeutic strategy for renal fibrosis $[8,9]$. In recent years, the replacement therapy of renal fibrosis by natural products has attracted the attention of many scholars [10]. Xiaoyan Shen et al. found that ginsenoside Rg1 ameliorated glomerular fibrosis during kidney aging by inhibiting the activation of NLRP3 inflammasome in SAMP8 mice [11]. This study explored the effect of $C$. flabellate (CF) extract on renal fibrosis in SAMP8 mice from the $\mathrm{Wnt} / \beta$-catenin/RAS pathway.

$\mathrm{CF}$ as a medicinal plant in China, was first recorded in the "Dian Nan Ben Cao." The whole plant is commonly used for treating dysentery, premature ejaculation, seminal vesicle disease, and kidney disease in ethnic minority areas of China [12]. At present, there are few reports about $\mathrm{CF}$ in modern research. The pharmacological studies in our laboratory found that CF extract had diuretic effects and ameliorated lipopolysaccharide/Dgalactosamine-induced liver failure and brain damage in rats $[13,14]$. And phytochemical studies on it revealed that phenylethanoid glycosides and flavonoids were the main chemical components of CF $[15,16]$. The aim of this study was to investigate the effect of CF extract on renal fibrosis in SAMP8 mice and to elucidate its possible mechanism, so as to provide experimental evidence for the treatment of kidney disease with CF described in ancient books.

\section{Materials and methods}

\section{Collection and extraction of the plant material}

"Yunnan Chinese Herbal Medicine" records that CF can be harvested throughout the year. The CF plants used in this experiment was harvested in Xixia County, Henan Province, China in September. It was identified by Professor Suiqing Chen from Henan University of Chinese Medicine, and the specimens were stored in the laboratory specimen library. The plants $(1 \mathrm{~kg})$ were refluxed with $50 \%$ ethanol $(3 \times 12 \mathrm{~L}$, each $1 \mathrm{~h})$, and the mixture was filtered with 16 layers of gauze. The combined filtrates were dried by rotary evaporation using a freeze drier. Finally, the percentage yield of $50 \%$ ethanol crude extract of CF was $15.5 \%$. The dried extract was kept in a fridge until further use. The supplementary materials list the relevant data on the ingredient designation of $\mathrm{CF}$ extract.

Another separation process previously reported was used in this study to obtain the water elution fraction, $20 \%$ ethanol elution fraction, $30 \%$ ethanol elution fraction, and $40 \%$ ethanol elution fraction from CF [13]. The $40 \%$ ethanol fraction was separated using Sephadex LH-20 and silica gel column and purified by semi-preparative high-performance liquid chromatography (HPLC) to finally obtain compounds such as SDC-0-14,16, SDC1-8, SDC-0-60 (p-hydroxybenzyl alcohol).

\section{Animals and administration}

Six-month-old male SAMP8 and senescence-accelerated mouse resistant 1 (SAMR1) mice from the First Affiliated Hospital of Tianjin University of Traditional Chinese Medicine (Tianjin, China) were used in this study. The animals were housed under controlled light (12-h light/ dark cycle), temperature $\left(23-25{ }^{\circ} \mathrm{C}\right)$, and humidity (45$55 \%)$ conditions and received a standard diet and water ad libitum. A total of 48 SAMP8 mice were divided into four experimental groups ( $n=12$ /group): SAMP8 model mice $(\mathrm{M})$, low-dose CF ethanol extract-treated SAMP8 mice (CF-L, $387.5 \mathrm{mg} / \mathrm{kg}$, intragastrically), medium-dose CF ethanol extract-treated SAMP8 mice (CF-M, $775 \mathrm{mg} /$ $\mathrm{kg}$, intragastrically), and high-dose $\mathrm{CF}$ ethanol extracttreated SAMP8 mice $(\mathrm{CF}-\mathrm{H}, 1550 \mathrm{mg} / \mathrm{kg}$, intragastrically). The mice in the SAMR1 control $($ Con, $n=10)$ and SAMP8 model groups $(M)$ were treated with physiological saline (0.9\%). All mice were treated orally for 1 month. All animal experiments were approved by the ethics committee of Henan University of Chinese Medicine and performed under the institutional guidelines (Henan, China Approval Number: HACTCM-2018009060-19). During the experiment, there was one mouse death in each of the Con and $M$ group, and two mice died in the CF-H group.

\section{Sample collection}

At the end of the experiment, the mice were housed individually in metabolic cages for 12-h urinary collection. The mice were then anesthetized with isoflurane, and blood samples were collected through retro-orbital bleeding. The kidneys from each mouse were then surgically removed and kept at $-80^{\circ} \mathrm{C}$ until the analyses.

\section{Cell culture and in vitro study}

NRK-52E cells purchased from the Cell Bank of the Chinese Academy of Sciences (Shanghai, China) were 
cultured to investigate the effect of CF extract on cell aging caused by D-galactose (D-gal, S11050, Yuanye, Shanghai, China). The NRK-52E cells were grown in Dulbecco's modification of Eagle's medium Dulbecco (DMEM, 12,100,046, Thermo Fisher, Massachusetts, USA) supplied with $10 \%$ fetal bovine serum in an incubator at $37{ }^{\circ} \mathrm{C}$ and in the presence of $5 \% \mathrm{CO}_{2}$. Cells were grown in 96-well plates or 6-well plates to $80-85 \%$ confluence and then treated with growth media containing different drug combinations: Media $+\mathrm{D}$-gal $(20 \mathrm{mg} /$ $\mathrm{mL})$, Media +D-gal +CF $(10,25,50,100 \mu \mathrm{g} / \mathrm{mL})$ or Media + D-gal + monomeric compound $(10 \mu \mathrm{M})$ respectively for $48 \mathrm{~h}$. Subsequently, the methyl thiazolyl tetrazolium (MTT) assay was used to detect cell viability, and $\beta$-galactosidase staining was used to observe cell senescence.

\section{Senescence-associated $\beta$-galactosidase staining}

Frozen kidneys from mice sliced into $10-\mu \mathrm{m}$-thick sections and NRK-52E cells were stained with senescenceassociated $\beta$-galactosidase (SA- $\beta$-gal, C0602, Beyotime Biotechnology, Shanghai, China) following the manufacturer's protocols.

\section{Histological analysis}

The kidney sections were fixed with $4 \%$ buffered paraformaldehyde, and 10- $\mu$ m-thick paraffin-embedded sections were stained with Masson's trichrome (G1006, Servicebio, Wuhan, China) and observed microscopically. The blue-colored areas in Masson's trichromestained sections were measured quantitatively from six randomly selected fields and analyzed by Image-Pro Plus 6.0 software.

\section{Biochemical measurements}

The serum and kidney homogenate samples were thawed to room temperature, and the levels of superoxide dismutase (SOD, CSB-E08556m, Wuhan Huamei, Wuhan, China), glutathione peroxidase (GSH-Px, A005-1-2, Nanjing Jiancheng, Nanjing, China), interleukin-1 $\beta$ (IL$1 \beta$, RK00006, ABclonal, Wuhan, China), tumor necrosis factor- $\alpha$ (TNF- $\alpha$, RK00027, ABclonal, Wuhan, China), urea nitrogen (C013-2-1, Nanjing Jiancheng, Nanjing, China), creatinine (Cr, C011-2-1, Nanjing Jiancheng, Nanjing, China) in mouse serum, total urinary protein (C035-2-1, Nanjing Jiancheng, Nanjing, China) in mouse urine and the expression levels of collagen type I (Col-I, MU30364, Bio-Swamp, Wuhan, China), $\alpha$-smooth muscle actin ( $\alpha$-SMA, MU30359, Bio-Swamp, Wuhan, China), fibronectin (FN, MU30179, Bio-Swamp, Wuhan, China) in mouse kidney tissues were measured sequentially according to the protocol of the assay kit manufacturer.

\section{Immunohistochemical analysis}

The immunohistochemical analysis was performed using the routine method [17]. The antibodies used included the following: Wnt4 (14371-1-AP, Proteintech, Chicago, USA), $\beta$-catenin (17565-1-AP, Proteintech, Chicago, USA), type 1 angiotensin II receptors (AGTR1, 253431-AP, Proteintech, Chicago, USA), p-nuclear factor erythroid 2-related factor 2 ( $p$-Nrf2, ab76026, Abcam, Cambridge, UK), $p$-c-Fos (ab27793, Abcam, Cambridge, UK), connective tissue growth factor (CTGF, GB11078, Servicebio, Wuhan, China). The sections were observed under a microscope (Olympus, Tokyo, Japan). Measure the area and integrated optical density (IOD) of the area with tan expression using Image-Pro Plus 6.0 software, and calculate the mean optical density (MOD, $\mathrm{MOD}=\mathrm{IOD} /$ area) for semi-quantitative analysis.

\section{Western blot analysis}

Western blot assay was conducted as described in a previous study [18]. Briefly, the kidney tissues were homogenized in lysis buffer and quantified using a Bradford Protein Assay Kit (AR0197, Boster Biological Technology, Wuhan, China). The homogenates were then subjected to sodium dodecyl sulfate-polyacrylamide gel electrophoresis, transferred to polyvinylidene fluoride membrane, and blocked in blocking buffer (4\% nonfat dry milk) for $90 \mathrm{~min}$. They were then incubated with primary antibodies (Wnt4; renin; AGTR1; p-Nrf2; p-c-Fos; Kelch like Ech associated protein 1 (Keap1, GB11847, Servicebio, Wuhan, China); $\beta$-actin (AC026, Abclonal, Wuhan, China); and glyceraldehyde-3-phosphate dehydrogenase (GAPDH, AC033, Abclonal, Wuhan, China)) overnight at $4^{\circ} \mathrm{C}$, followed by incubation with an appropriate fluorescence-conjugated secondary antibody for $1 \mathrm{~h}$ at room temperature. The proteins of interest were scanned with an Odyssey IR scanner (LI-COR Biosciences, Nebraska, USA), and the signal intensities were quantified using Image Studio software. The protein levels were normalized against the $\beta$-actin or GAPDH.

\section{UPLC-Q-TOF-MS analysis for kidney samples}

The renal tissues were weighed and homogenized in ice-cold physiological saline $(\mathrm{w} / \mathrm{v}=1: 1)$. Then, $1 \mathrm{~mL}$ of acetonitrile was added to $200 \mu \mathrm{L}$ of tissue homogenate samples, followed by ultrasonic extraction for $30 \mathrm{~min}$. The extract was centrifuged at $12,000 \mathrm{~g}$ and $4{ }^{\circ} \mathrm{C}$ for $10 \mathrm{~min}$. The supernatant was taken into the vial for analysis. Chromatographic separation was carried out on ultraperformance liquid chromatography (UPLC) (Dionex UltiMate 3000 System, Thermo Scientific, Massachusetts, USA), with the LC system comprising an Acclaim RSLC $120 \mathrm{C}_{18}$ column $(2.2 \mu \mathrm{m}$, 
$2.1 \times 100 \mathrm{~mm}$; Thermo Scientific). The mobile phase consisted of solvent A (acetonitrile) and water with $0.1 \%$ formic acid (B). The separation was performed by gradient elution as follows: $10-70 \%$ A from 0 to $3 \mathrm{~min}, 70-78 \%$ A from 4 to $13 \mathrm{~min}, 78-90 \%$ A from 14 to $15 \mathrm{~min}, 90 \%-10 \% \mathrm{~A}$ from 15 to $16 \mathrm{~min}$, and $10 \% \mathrm{~A}$ from 16 to $20 \mathrm{~min}$. The injection volume of the test sample was $2 \mu \mathrm{L}$. The mass spectrometry (MS) analysis was performed by using an ESI source under the following conditions: the capillary voltage in the positive mode was $3.5 \mathrm{kV}$, and the capillary voltage in the negative mode was $3.2 \mathrm{kV}$. The pressure of the nebulizer was $2.0 \mathrm{bar}$, the temperature of the dry gas was $230{ }^{\circ} \mathrm{C}$, and the flow rate was $8 \mathrm{~L} / \mathrm{min}$.

\section{Statistical analysis}

The acquired raw data from ultra-performance liquid chromatography coupled to quadrupole time-of-flight mass spectrometry (UPLC-Q/TOF-MS) analysis were first preprocessed using profile analysis (version 2.1, Bruker, Germany). The "bucket table" was obtained and imported into the SIMCA-P software (version13.0 Umetrics AB, Sweden) for principal component analysis (PCA). Other results were presented as mean \pm standard deviation (SD). The data were processed using IBM SPSS Stastic 26.0. The normal distribution of the data used the Levene test, which required the average value, $P>0.05$; the one-way analysis of variance was used for comparison between groups. A $P$ value less than 0.05 was considered statistically significant.

\section{Results}

\section{CF improved the aging and fibrosis of the kidneys in SAMP8 mice}

To investigate the effect of CF on the kidneys of SAMP8 mice, SA- $\beta$-gal activity was measured in the kidneys. In Fig. 1a, The SA- $\beta$-gal activity in kidney tissues significantly increased in the $M$ group (blue enhancement). Administration of CF-L and CF-M significantly inhibited $\beta$-galactosidase activity in the kidneys of SAMP8 mice while the improvement by $\mathrm{CF}-\mathrm{H}$ was not significant. Also, the kidney index of the model group was significantly lower than that of the control group (Fig. 1b, $P<0.05)$. After treatment with CF-M, the kidney index of SAMP8 mice were improved $(P<0.01)$. Next, the results of Masson's trichrome staining (Fig. 1c, $P<0.01$ ) and the expression of fibrosis indicators Col-I, $\alpha-S M A$, and $\mathrm{FN}$ revealed that $\mathrm{M}$-group mice had more severe renal fibrosis than Con-group mice, and CF-L and CF-M significantly attenuated renal fibrosis in SAMP8 mice (Fig. 1e-g, $P<0.01$ ).
CF improved renal function, oxidative stress and inflammation levels in SAMP8 mice

Besides, the renal function indicators of each group of mice were tested, which included urine volume in mice for $12 \mathrm{~h}, \mathrm{Cr}$ and urea nitrogen levels in the serum, and urine protein levels. As shown in Fig. 2a-d, the urine protein, serum $\mathrm{Cr}$ and serum urea nitrogen levels of the model group were significantly higher than those of the control group (Fig. 2a, $P<0.01$ ), while the urine volume of the model group was significantly lower than that of the control group (Fig. $2 \mathrm{~b}-\mathrm{d}, \mathrm{P}<0.01$ ). CF supplementation down-regulated the levels of urine protein, serum $\mathrm{Cr}$ and urea nitrogen in the model group (Fig. $2 \mathrm{~b}-\mathrm{d}, P<0.05$ or $P<0.01)$ while increasing the urine output of model mice. Collectively, the results indicated that SAMP8 mice had kidney damage associated with aging, and treatment with CF effectively ameliorated this injury. Next, whether CF beneficially modulated oxidative stress and inflammation in the kidneys of rapidly aging mice was explored. As shown in Fig. 2e-h, the levels of SOD and GSH-Px in the kidneys of the $M$ group were significantly lower than those of the Con group, and the levels of IL-1 $\beta$ were significantly higher than those of the Con group. After CF treatment, the above indicators have been improved, especially the improvement effect of CF-L and CF-M is better.

\section{CF activated the Nrf2 pathway in the kidney of SAMP8 mice}

The expression level of $p$-Nrf2 was measured in kidney tissues to investigate whether the Nrf2 pathway was involved in the protective effect of CF (Fig. 3a-c) in the present study. The expression level of $p$-Nrf2 significantly decreased in the SAMP8 group alone $(P<0.01$, Fig. $3 \mathrm{c})$, and the downregulation was reversed to some extent by the treatment of CF crude extract. There was no significant change in the expression level of Keap1 among the groups (Fig. 3b and d). Further, the expression level of $p$-c-Fos was detected and analyzed by immunohistochemical and Western blot analyses. It was found that the expression level of $p$-c-Fos in the kidneys of mice in the $M$ group was significantly higher than that in the Con group; while CF treatment significantly reduced the expression levels of $p$-c-Fos in the kidneys of the mice (Fig. 3a-b and e).

\section{CF attenuated Wnt/ $\beta$-catenin/RAS signaling activation in the kidney of SAMP8 mice}

In order to further explored the potential mechanism of CF exerting anti-fibrosis effect. The localization of protein was performed using immunohistochemistry. As shown in Fig. 4a, the expression level of Wnt4 and 


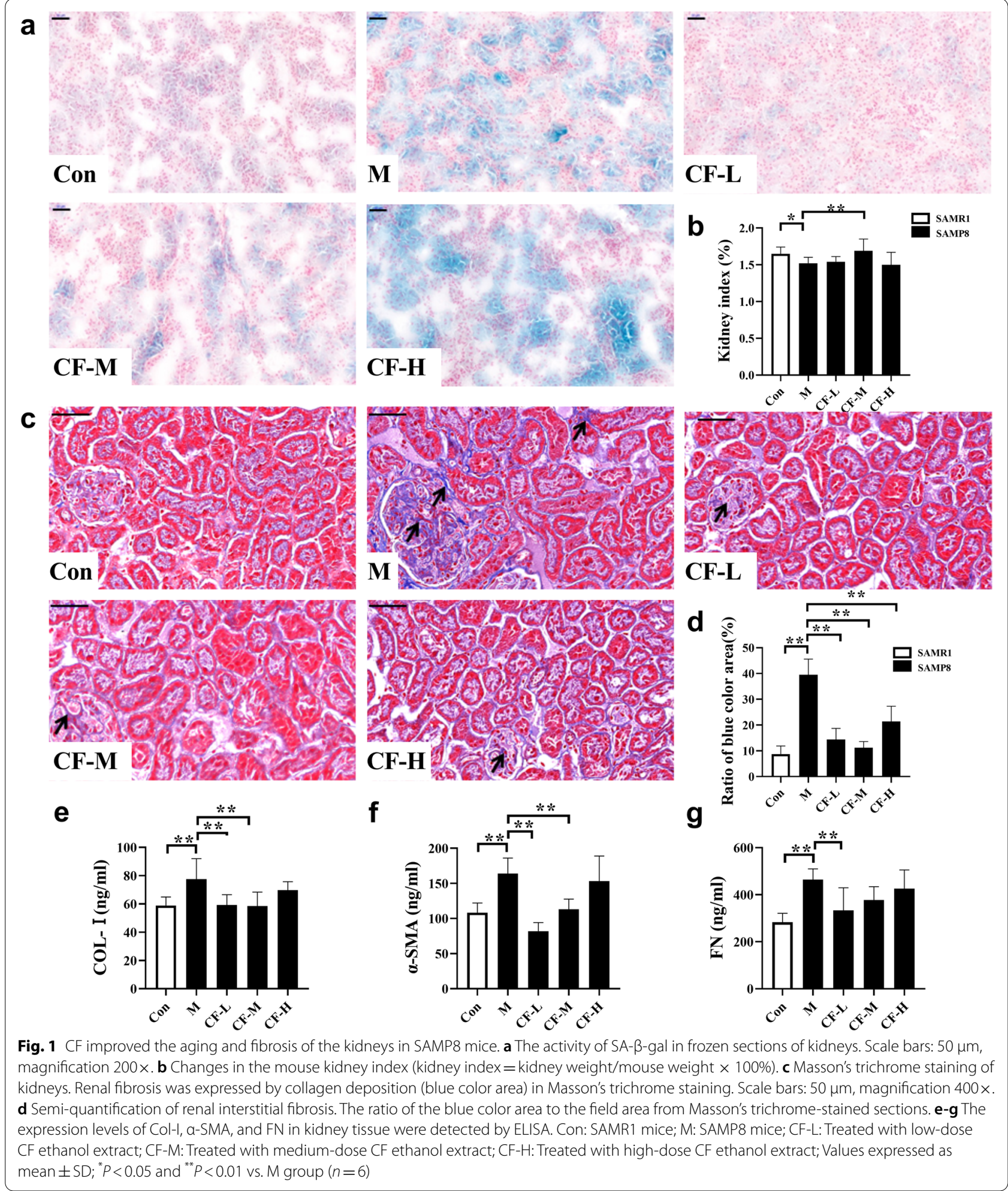

$\beta$-catenin was induced predominantly in renal tubular cells. Similar results were observed when AGTR1, the downstream pathway targets of Wnt signaling, were assessed (Fig. 4a). After that, the protein expression level was quantified, and the results showed that Wnt4, $\beta$-catenin, renin, and AGTR1 proteins were 


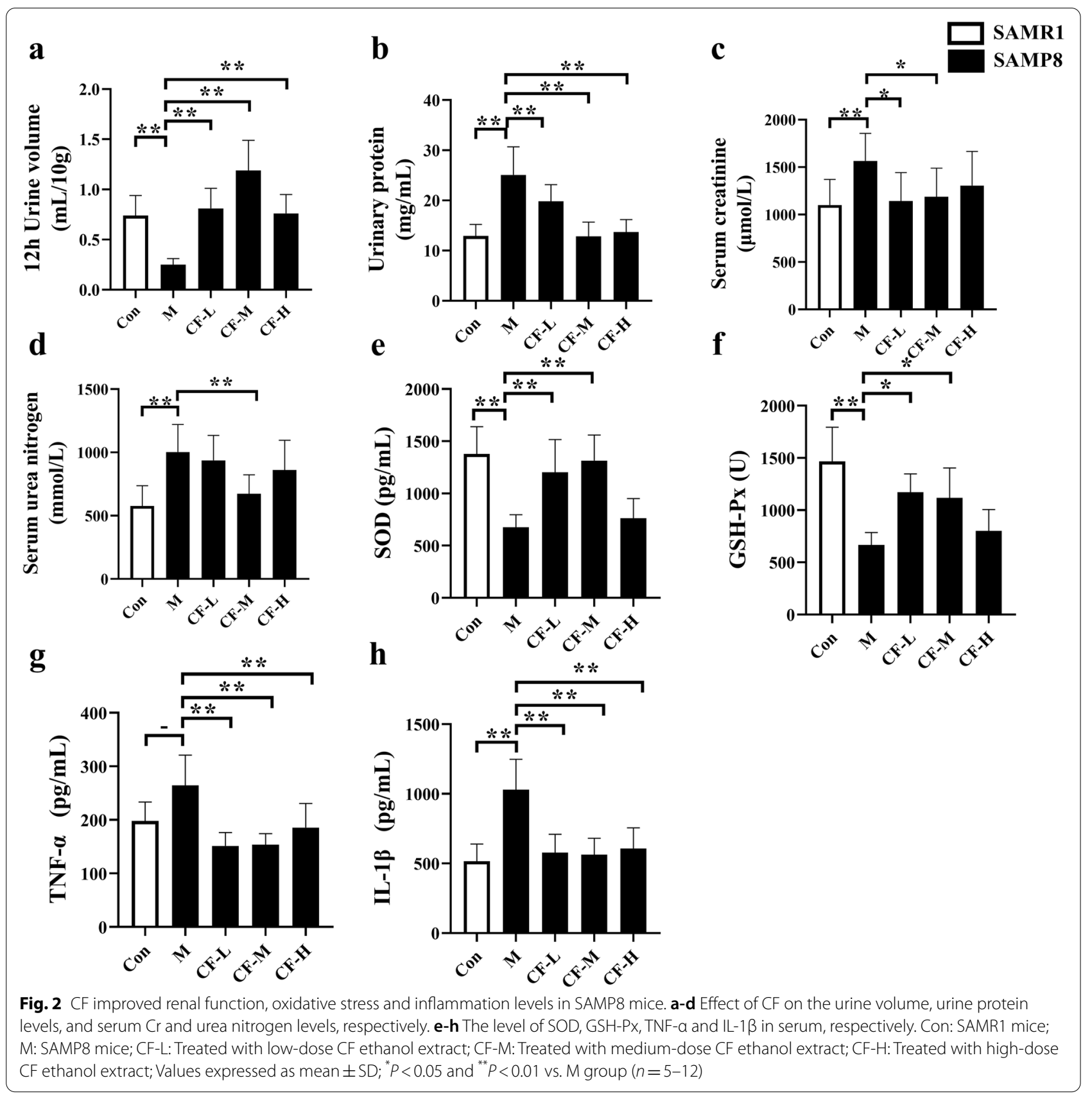

accumulated in the kidney tissue of mice in the model group, whereas CF significantly inhibited those alterations (Fig. 4b-f ). Furthermore, the expression level of CTGF was also located and quantitatively analyzed. Consistent with the aforementioned results, CTGF was mainly expressed in the renal tubules, and CF markedly inhibited the activity of CTGF (Fig. $4 \mathrm{~d}$ g).
CF and its compounds inhibited the senescence of NRK-52E cells induced by $\mathrm{D}$-gal

D-gal-induced NRK-52E cells were used to screen certain active ingredients in CF, to determine the material basis for the treatment of senile kidney by the extract of CF. The results showed that the CF enhanced cell viability in a dose-dependent manner, and the compounds 


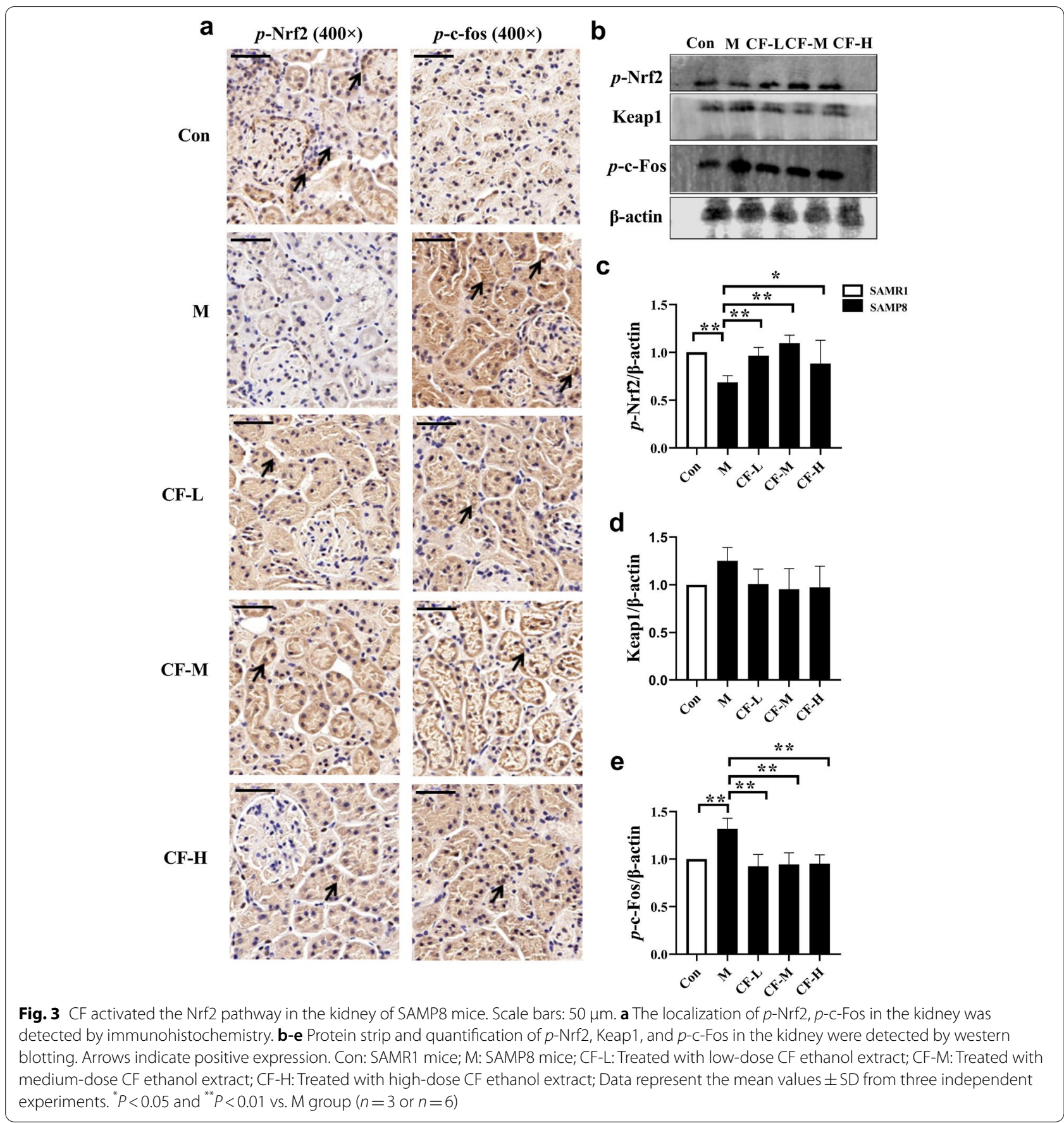

SDC-0-14,16 and SDC-1-8 isolated from CF had better effects on cell proliferation. Besides, $25 \mu \mathrm{g} / \mathrm{mL} \mathrm{CF}$ and the compounds SDC-0-14,16 and SDC-1-8 all displayed the effect of inhibiting $\beta$-galactosidase activity (Fig. 5).

\section{CF improved PCA analysis of kidney tissue in SAMP8 mice} Principal components analysis (PCA) was performed to explore the effects of CF on SAMP8 mice. As shown in
Fig. 6a, there was an obvious grouping between the control and model groups $(\mathrm{R} 2 \mathrm{X}=0.542 ; \mathrm{Q} 2=0.38)$, suggesting that the endogenous metabolites of SAMP8 mice were different from SAMR1 mice, which causes them to deviate from the SAMR1 group. As shown in Fig. 6b, the different CF groups also clustered into different classes from the control and model groups, and the CF groups moved closer to the control group, indicating that the 


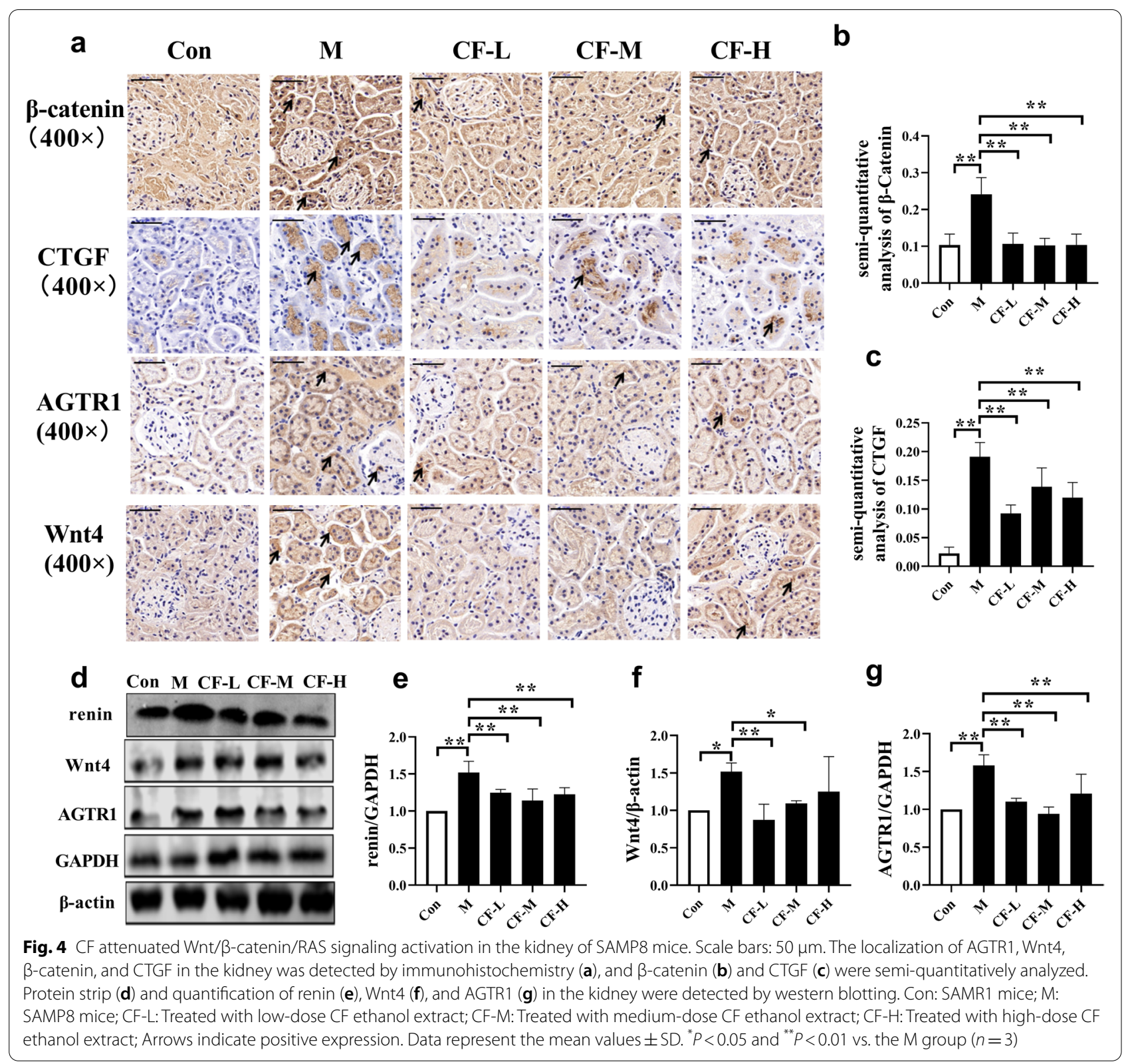

endogenous metabolites of the CF-intervened SAMP8 mice were similar to those of the SAMR1 mice.

\section{Discussion}

Aging plays an important role in the progression of CKD [19]. As CKD fibrosis progresses, senescent cells express and secrete pro-fibrotic factors (TGF- $\beta$, CTGF, and so forth) and pro-inflammatory factors (IL-1 $\beta$, IL-6, TNF$\alpha$, and so forth), which are senescence-associated secretory phenotype factors, thereby accelerating renal fibrosis $[20,21]$. At present, traditional Chinese medicine has achieved good results in treating renal fibrosis with fewer side effects [22]. This study explored the interventional effects of CF extract on renal fibrosis in SAMP8 mice.

The mouse strain selected for this study was SAMP8, which was developed based on the lifespan, senescence, and pathological phenotypic grading scores of $\mathrm{AKR} / \mathrm{J}$ mice and was an accelerated aging model solely of genetic origin [23, 24]. Studies have shown that the changes in the renal pathology of SAMP8 mice ( 9 months) include tubulointerstitial fibrosis and focal segmental glomerulosclerosis [25]. And it was found that renal fibrosis in SAMP8 mice was age-related [6]. Therefore, we tested the activity of $\beta$-galactosidase and 

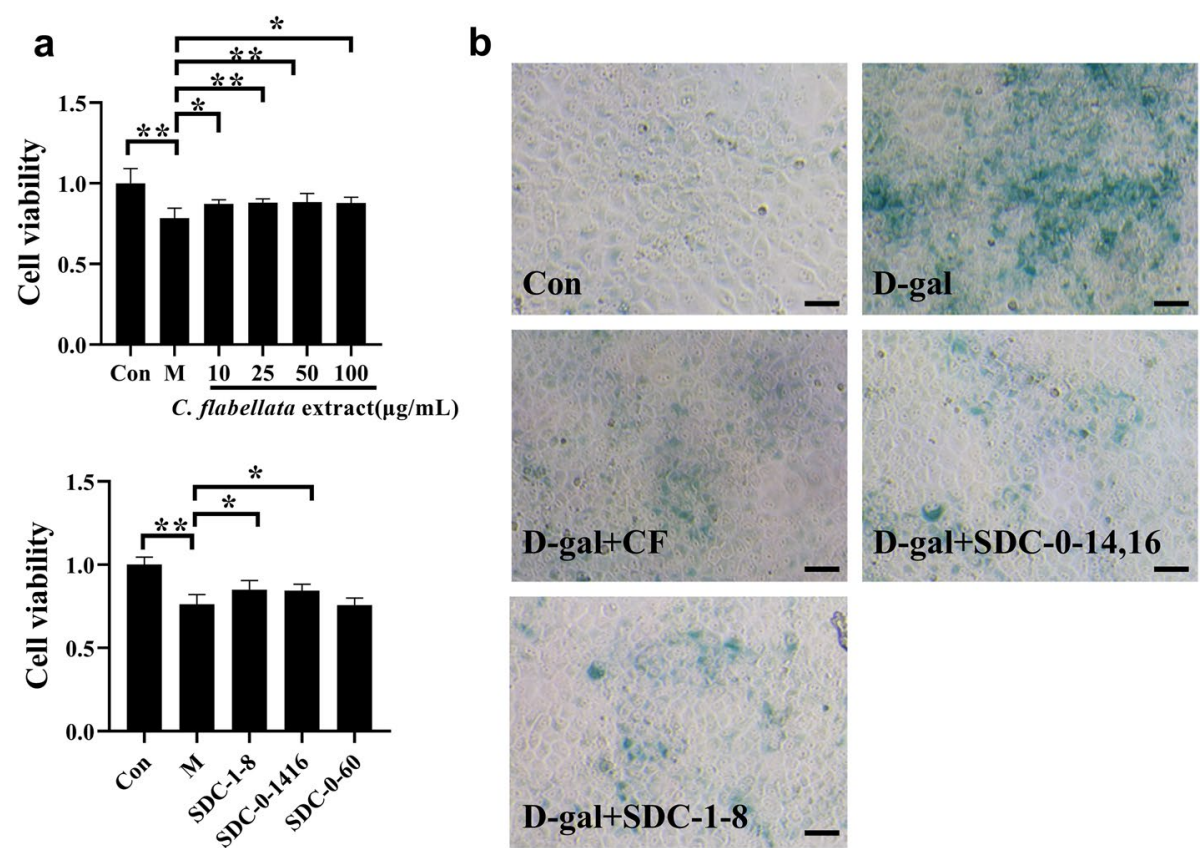

Fig. 5 CF and its compounds inhibited the senescence of NRK-52E cells induced by D-gal. a Detection of cell viability of CF and its chemical components. b $\beta$-galactosidase staining of NRK-52E cells. Scale bars: 50 m. CF: C. flabellate extract; D-gal: D-galactose; SDC-0-14,16: 3,4-dihydroxyphenylethanol; SDC-1-8: (3,4-dihydroxyphenylethanol-8-O-[4-O-trans-caffeoyl- $\beta$-D-apiofuranosyl-(1 $\rightarrow 3$ )- $\beta$-D-glucopyranosyl $(1 \rightarrow 6)]-\beta$-D-glucopyranoside; SDC-0-60: $p$-hydroxybenzyl alcohol. Data represent the mean values \pm SD from three independent experiments. ${ }^{*} P<0.05$ and ${ }^{* *} P<0.01$ vs. the $D$-gal group

the level of renal fibrosis in SAMP8 mice, and found that CF-L and CF-M improved kidney fibrosis and $\beta$-galactosidase activity in SAMP8 mice (Fig. 1). And we also tested the urine output of the mice. Consistent with previously published studies indicating that $\mathrm{CF}$ obtained by two different processes had diuretic effects [13], the extract significantly increased the urine volume of SAMP8 mice. Also, the present study showed that supplementation with CF improved the renal function, the activity of antioxidant enzymes, and levels of inflammatory factors in SAMP8 mice (Fig. 2).

The Keap1-Nrf2 system gained the attention of many scholars in recent years due to its antioxidant and antiinflammatory properties. Its pharmacological potential in treating kidney diseases had been extensively studied in nonclinical and clinical studies [26]. Nrf2 is a master transcriptional regulator for genes related to redox status and antioxidant effects [27]. Studies have shown that phosphorylation is required for Nrf2 activation and target gene induction [28]. Activating Nrf2 improved CKD progression by preventing oxidative stress and maintaining cellular redox homeostasis [29]. As a key transcription factor, Nrf2 plays a crucial role in defense against oxidative stress by regulating its downstream antioxidants and detoxification enzymes [30]. Kim et al. reported that resveratrol, as a potent Nrf2 activator, ameliorated aging-related progressive renal injury [31]. In the present study, CF improved the attenuation of Nrf2 in the kidney of SAMP8 mice without affecting the expression level of Keap1, suggesting that $\mathrm{CF}$ crude extract might improve oxidative damage in the kidneys by activating the Nrf2 pathway (Fig. 3).

Renal fibrosis is characterized by excessive extracellular matrix (ECM) deposition leading to the formation of scars in the renal parenchyma [32]. Transforming growth factor- $\beta$ (TGF- $\beta$ ) is thought to be a key cytokine in fibroblast overactivation [33, 34]. Of course, targeting only the TGF- $\beta$ signaling pathway is insufficient to reduce renal fibrosis. Some studies indicated that CTGF, Wnt/ $\beta$-catenin, renin-angiotensin system, oxidative stress, and so forth, were implicated in renal fibrosis [35-37]. Wnt $/ \beta$-catenin is an evolutionarily conserved signaling pathway involved in the regulation of tissue homeostasis, organ development, and injury repair [38]. Cisternas et al. discussed the pro-fibrotic effect of Wnt signaling in both skeletal muscle and kidney [39]. Mounting evidence established that the $\mathrm{Wnt} / \beta$-catenin signaling pathway plays a crucial role in regulating the development and progression of renal fibrotic lesions following injury [40-42]. The Wnt/ $\beta$-catenin signal is relatively silent in the kidneys of healthy adults and is activated once the kidneys are subjected to various kinds of damages [43]. 

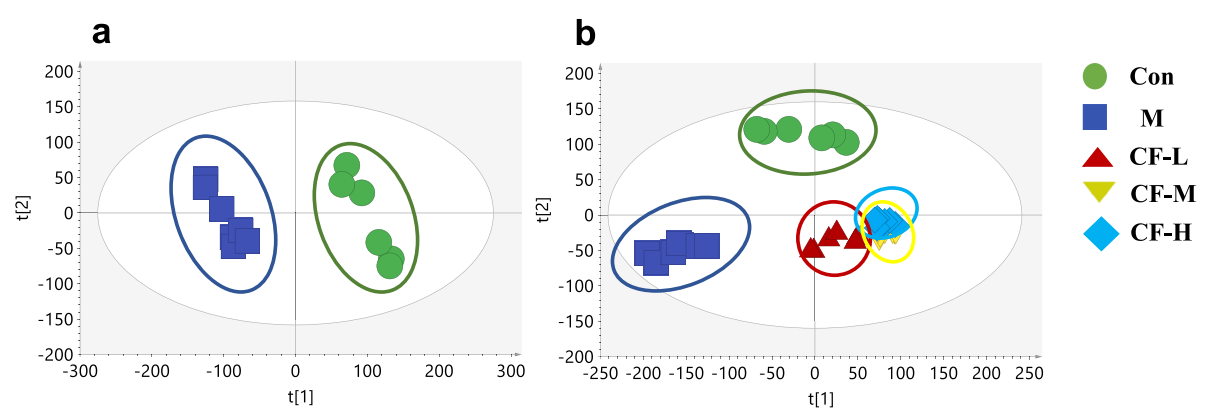

Fig. 6 PCA score plot of kidney homogenate extract in positive modes. a PCA score plot for the Control and M groups $(R 2 X=0.542, Q 2=0.38)$; b PCA score plot for all groups $(R 2 X=0.63, Q 2=0.489)$. Con: SAMR1 mice; M: SAMP8 mice; CF-L: Treated with low-dose CF ethanol extract; CF-M: Treated with medium-dose CF ethanol extract; CF-H: Treated with high-dose CF ethanol extract;

In mammals, the Wnt family has at least 19 family members critical for kidney development. And at least 15 of these family members are differentially upregulated in the aging kidney, including Wnt4 [6]. The results of the present study showed that the expression level of Wnt4 protein in the kidneys of SAMP8 mice was significantly higher than that in SAMR1 mice, which was verified by both Western blot and immunohistochemical analyses (Fig. 4a, d and f). Coincidentally, the expression level of $\beta$-catenin protein was also upregulated. Also, both Wnt4 and $\beta$-catenin were expressed in renal tubular epithelial cells, as revealed by the immunohistochemical analysis (Fig. 4a and f). Wnt/ $\beta$-catenin elicited renal fibrosis by inducing multiple fibrogenic genes such as RAS components, matrix metalloproteinase-7 (MMP-7), plasminogen activator inhibitor 1 (PAI-1), and Snail1 [37]. Zhou et al. described $\mathrm{Wnt} / \beta$-catenin as the major upstream regulator, which controls the expression of all tested RAS components in the kidneys [44]. Zhou et al. used a 5/6 nephrectomy $(5 / 6 \mathrm{NX})$ rat model to show that the expression levels of major components of RAS in the brain and kidneys, such as angiotensinogen, angiotensin-converting enzyme, and angiotensin II AT1-receptor, was significantly upregulated. The upregulated expression level was inhibited by a central blocker of Wnt, which was an adeno-associated virus vector overexpressing the $D K K 1$ gene [45]. Similarly, the present study found that AGTR1 was still expressed in the renal tubular epithelium, and the expression levels of renin and AGTR1 in the kidney tissues of SAMP8 mice were significantly greater than those in SAMR1 mice (Fig. 4a and d g). These results indicated that the Wnt/ $\beta$-catenin/RAS signaling pathway was activated in the kidneys of SAMP8 mice, and the CF effectively inhibited the activation of this pathway.

CTGF exerts multiple biological functions, including promoting mitosis of chemotactic cells, inducing adhesion, and promoting cell proliferation and ECM synthesis $[35,46]$. CCN2 (CTGF) modulated Wnt signaling by binding to low-density lipoprotein receptor-related protein 5/6 (LRP5/6) to further mediate fibrosis [39, 47]. Other studies used gene silencing to discover and confirm that Nrf2 regulated the Wnt pathway by regulating the expression level of CTGF, affecting renal interstitial disease. Also, Nrf2 regulated the CTGF transcription level mainly via CTGF transcriptional regulator c-Fos [48]. The immunohistochemical analysis revealed that CTGF and $p$-c-Fos were expressed mainly in renal tubular epithelial cells, and $p$-c-Fos was also distributed in glomeruli. The quantitative analysis showed that the expression levels of both proteins was inhibited by CF (Figs. 3b and $\mathrm{e}$ and $4 \mathrm{a}$ and c). Finally, the PCA analysis was used to further verify that CF improved kidney damage in SAMP8 mice (Fig. 6). The present study provided evidence that the CF not only activated Nrf2 signaling but also relied on Nrf2 to balance oxidative stress and inflammation, inhibited Wnt/ $\beta$-catenin/RAS signaling, and improved kidney aging and renal fibrogenesis. However, we also found inconsistent improvement in the effect of CF on Masson and SA- $\beta$-gal staining in this experiment. It is speculated that this may be due to the inconsistent progression of renal aging and renal fibrosis in SAMP8 mice. The degree of renal aging in the mice in this experiment may be more serious than fibrosis. Therefore, the ameliorating effect of $\mathrm{CF}-\mathrm{H}$ on renal aging in SAMP8 mice was not as obvious as that of renal fibrosis.

As a reducing monosaccharide, D-galactose is widely used in various age-related diseases in vivo and in vitro. D-gal was found to cause senescence and injury in NRK$52 \mathrm{E}$ cells [49], induce senescence of human kidney proximal tubular epithelial cells (HKC-8 cells), and increase the expression levels of two renal fibrosis marker proteins FN and $\alpha$-SMA [6]. In this study, D-gal was used to induce NRK-52E cells in vitro. Using MTT assay and $\beta$-galactosidase staining to detect the compounds isolated from CF, it was found that SDC-0-14,16 and SDC1-8 enhanced the cell viability induced by $\mathrm{D}$-gal and 
inhibited D-gal induced cell senescence (Fig. 5). These suggested that SDC-0-14,16 and SDC-1-8 may be the material basis for CF to delay kidney aging. SDC-1-8 is one of the phenylethanoid glycosides. phenylethanoid glycosides were found to extend the life span of Caenorhabditis Elegans [50], with anti-aging, [51] and neuroprotective effects [52-54]. These provide a direction for our follow-up study on the pharmacological effects of CF.

\section{Conclusions}

In conclusion, in vivo studies showed that CF reduced renal fibrosis in elderly mice. Some potential active ingredients were found in in vitro experiments. These findings provided pharmacological support for treating kidney disease using $\mathrm{CF}$ and a direction for further research on the active ingredients of $\mathrm{CF}$.

\begin{abstract}
Abbreviations
CKD: Chronic kidney disease; C. flabellate: Corallodiscus flabellata B. L. Burtt; RAS: Renin-angiotensin system; SDC-0-14, 16: 3,4-dihydroxyphenylethanol; SDC-1-8: (3,4-dihydroxyphenylethanol-8-O-[4-O-trans-caffeoyl- $\beta$-Dapiofuranosyl-( $(\rightarrow 3)-\beta$-D-glucopyranosyl $(1 \rightarrow 6)]-\beta-D$-glucopyranoside; SDC-0-60: p-hydroxybenzyl alcohol; HPLC: High-performance liquid chromatography; NRK-52E cells: normal rat kidney epithelial cells; DMEM: Dulbecco's modification of Eagle's medium Dulbecco; D-gal: D-galactose; SAMP8: Senescence-accelerated mouse-prone 8; SAMR1: Senescenceresistant mouse-prone 1; SOD: superoxide dismutase; IL: interleukin; TNF-a: tumor necrosis factor-a; Col-I: Collagen type I; a-SMA: a-smooth muscle actin; FN: fibronectin; Cr: creatinine; GSH-Px: glutathione peroxidase; AGTR1: type 1 angiotensin II receptors; CTGF: connective tissue growth factor; p-Nrf2: p-nuclear factor erythroid 2-related factor 2; Keap 1: Kelch-like Ech-associated protein-1; GAPDH: glyceraldehyde-3-phosphate dehydrogenase; UPLC-Q/TOFMS: ultra-performance liquid chromatography coupled to quadrupole timeof-flight mass spectrometry; PCA: principal component analysis; SD: standard deviation; SA- $\beta$-gal: senescence-associated $\beta$-galactosidase; ECM: excessive extracellular matrix; TGF- $\beta$ : transforming growth factor- $\beta$; HKC-8 cells: Human kidney proximal tubular epithelial cells.
\end{abstract}

\section{Supplementary Information}

The online version contains supplementary material available at https://doi. org/10.1186/s12906-022-03535-y.

\section{Additional file 1.}

Additional file 2.

Additional file 3.

\section{Acknowledgements}

Not applicable.

\section{Statements}

A preprint version of this article is available on Research Square [55].

\section{Authors' contributions}

$B C, Y S$, and $Y W$ performed the experiments. BC, BZ, and $R X$ analyzed data, plotted the graphs for figures. $M Z, B C, Y H, X Z$, and WF participated in discussions. $B C$ and $M Z$ drafted the manuscript. All authors read and approved the final manuscript.

\section{Funding}

This work was supported in part by the National Key Research and Development Project (2019YFC1708802) to XZ for animal experiments, by the Major
Science and Technology Projects in Henan Province (171100310500) to WF for cell experiments, by the Henan province high-level personnel special support "Zhong Yuan One Thousand People Plan" - Zhongyuan Leading Talent (ZYQR201810080) to XZ for other experimental consumables. The results described in this paper were part of student thesis.

\section{Availability of data and materials}

All data generated or analyzed during this study are included in this published article. Raw data of this study can be asked to the corresponding author if needed.

\section{Declarations}

\section{Ethics approval and consent to participate}

All experimental procedures were approved by the Research Ethics Committee of Henan University of Chinese Medicine. These data were not subjected to clinical trial or involving human studies. It is confirmed that all methods have been performed in accordance with the relevant guidelines and regulations. Also, it is confirmed that the study was carried out in compliance with the ARRIVE guidelines. The CF plants were sought from the Xixia County, Henan province, China. The collection of the wild plant samples complied with the Chinese Medicine Resources department's guidelines at the Henan University of Chinese Medicine and were stored in the specimen bank (No. 20171021 A).

\section{Consent for publication}

Not applicable.

\section{Competing interests}

The authors declare that they have no competing interests.

\section{Author details}

${ }^{1}$ Henan University of Chinese Medicine, 450046 Zhengzhou, China. ${ }^{2}$ The Engineering and Technology Center for Chinese Medicine Development of Henan Province, 450046 Zhengzhou, China. ${ }^{3}$ School of Pharmacy, Henan University of Chinese Medicine, 156 Jinshui East Road, 450046 Zhengzhou, China.

Received: 10 October 2021 Accepted: 22 February 2022

Published online: 28 February 2022

\section{References}

1. Wang V, Vilme H, Maciejewski ML, Boulware LE: The Economic Burden of Chronic Kidney Disease and End-Stage Renal Disease. Semin Nephrol 2016, 36(4):319-330.

2. Kitai Y, Nangaku M, Yanagita M: Aging-Related Kidney Diseases. Contrib Nephrol 2021, 199:266-273.

3. Collaborators GBDCoD: Global, regional, and national age-sex specific mortality for 264 causes of death, 1980-2016: a systematic analysis for the Global Burden of Disease Study 2016. Lancet 2017, 390(10100):1151-1210.

4. Paniagua-Sierra JR, Galvan-Plata ME: Chronic kidney disease. Revista medica del Instituto Mexicano del Seguro Social 2017, 55(Suppl 2):S116-117.

5. Xu J, Zhou L, Liu Y: Cellular Senescence in Kidney Fibrosis: Pathologic Significance and Therapeutic Strategies. Frontiers in pharmacology 2020, 11:601325.

6. Miao J, Liu J, Niu J, Zhang Y, Shen W, Luo C, Liu Y, Li C, Li H, Yang P et al: Wnt/beta-catenin/RAS signaling mediates age-related renal fibrosis and is associated with mitochondrial dysfunction. Aging cell 2019, 18(5):e13004

7. Zhou L, LiY, Hao S, Zhou D, Tan RJ, Nie J, Hou FF, Kahn M, Liu Y: Multiple genes of the renin-angiotensin system are novel targets of Wnt/betacatenin signaling. Journal of the American Society of Nephrology: JASN 2015, 26(1):107-120.

8. Li SS, Sun Q, Hua MR, Suo P, Chen JR, Yu XY, Zhao YY: Targeting the Wnt/ beta-Catenin Signaling Pathway as a Potential Therapeutic Strategy in Renal Tubulointerstitial Fibrosis. Frontiers in pharmacology 2021, 12:719880. 
9. Zhou G, Li J, Zeng T, Yang P, Li A: The regulation effect of WNT-RAS signaling in hypothalamic paraventricular nucleus on renal fibrosis. Journal of nephrology 2020, 33(2):289-297.

10. Chen DQ, Hu HH, Wang YN, Feng YL, Cao G, Zhao YY: Natural products for the prevention and treatment of kidney disease. Phytomedicine: international journal of phytotherapy and phytopharmacology 2018, 50:50-60.

11. Shen X, Dong X, Han Y, Li Y, Ding S, Zhang H, Sun Z, Yin Y, Li W, Li W: Ginsenoside $\mathrm{Rg} 1$ ameliorates glomerular fibrosis during kidney aging by inhibiting NOX4 and NLRP3 inflammasome activation in SAMP8 mice. Int Immunopharmacol 2020, 82:106339.

12. Bai $Z F$, Wang $X Q$, Xiao $P G$, Liu Y: Investigation of Gesneriaceae ethnic medicinal plants in Guangxi Zhuang Autonomous Region. Zhong yao cai $=$ Zhongyaocai $=$ Journal of Chinese medicinal materials 2012, 35(1):20-23.

13. Kan $Y$, Zeng $M$, Zhang $B$, Li B, Wang $S$, Wang $Y, X u R$, Wu Y, Zheng $X$, Feng W: Corallodiscus flabellata B.L. Burtt Extracts Stimulate Diuretic Activity and Regulate the Renal Expression of Aquaporins. Evidencebased complementary and alternative medicine: eCAM 2020, 2020:6020817.

14. Li B, Zeng M, Zhang B, Kan Y, Wang S, Wang Y, Wu Y, Xu R, Feng W, Zheng X: Corallodiscus flabellata B. L. Burtt extract alleviates lipopolysaccharide/D-galactosamine-induced acute liver failure and brain injury by inhibiting oxidative stress, apoptosis, and inflammation. Iran J Basic Med Sci 2020, 23(11):1445-1452.

15. Feng WS, Zheng XK, Liu YB, Kuang HX: Three new C-flavonoids from Corallodiscus flabellata. Journal of Asian natural products research 2007, 9(1):85-89.

16. Feng WS, Zheng XK, Liu YB, Li J: Isolation and structural identification of C-glycosylflavones from Corallodiscus flabellata. Yao xue xue bao = Acta pharmaceutica Sinica 2004, 39(2):110-115.

17. Mao Q, Chen C, Liang H, Zhong S, Cheng X, Li L: Astragaloside IV inhibits excessive mesangial cell proliferation and renal fibrosis caused by diabetic nephropathy via modulation of the TGF-beta1/Smad/miR192 signaling pathway. Experimental and therapeutic medicine 2019, 18(4):3053-3061.

18. Li M, Zeng M, Zhang ZG, Zhang B, Zhang J, Zheng X, Feng W: Lepidiumuridine A: A New Natural Uridine Derivative as a Phytoestrogen Isolated from the Seeds of Lepidium apetalum Willd. Evidence-based complementary and alternative medicine: eCAM 2018, 2018:2813465.

19. Valentijn FA, Falke LL, Nguyen TQ, Goldschmeding R: Cellular senescence in the aging and diseased kidney. Journal of cell communication and signaling 2018, 12(1):69-82.

20. Knoppert SN, Valentijn FA, Nguyen TQ, Goldschmeding R, Falke LL: Cellular Senescence and the Kidney: Potential Therapeutic Targets and Tools. Frontiers in pharmacology 2019, 10:770.

21. Docherty MH, O'Sullivan ED, Bonventre JV, Ferenbach DA: Cellular Senescence in the Kidney. Journal of the American Society of Nephrology: JASN 2019, 30(5):726-736.

22. Shen YL, Wang SJ, Rahman K, Zhang LJ, Zhang H: Chinese Herbal Formulas and Renal Fibrosis: An Overview. Curr Pharm Des 2018, 24(24):2774-2781.

23. Takeda T, Matsushita T, Kurozumi M, Takemura K, Higuchi K, Hosokawa M: Pathobiology of the senescence-accelerated mouse (SAM). Experimental gerontology 1997, 32(1-2):117-127.

24. Takeda T, Hosokawa M, Higuchi K, Hosono M, Akiguchi I, Katoh H: A novel murine model of aging, Senescence-Accelerated Mouse (SAM). Archives of gerontology and geriatrics 1994, 19(2):185-192.

25. Chaohui SXCXSYYMYHZ: The pathological characteristic of aging kidney in senescence accelerated mouse/prone 8. Chinese Journal of Gerontology 2010, 30(03):327-329.

26. Yamawaki K, Kanda H, Shimazaki R: Nrf2 activator for the treatment of kidney diseases. Toxicology and applied pharmacology 2018, 360:30-37.

27. Nezu M, Suzuki N, Yamamoto M: Targeting the KEAP1-NRF2 System to Prevent Kidney Disease Progression. American journal of nephrology 2017, 45(6):473-483.

28. Bryan HK, Olayanju A, Goldring CE, Park BK: The Nrf2 cell defence pathway: Keap1-dependent and -independent mechanisms of regulation. Biochemical pharmacology 2013, 85(6):705-717.

29. Choi BH, Kang KS, Kwak MK: Effect of redox modulating NRF2 activators on chronic kidney disease. Molecules 2014, 19(8):12727-12759.
30. Ruiz S, Pergola PE, Zager RA, Vaziri ND: Targeting the transcription factor $\mathrm{Nrf2}$ to ameliorate oxidative stress and inflammation in chronic kidney disease. Kidney international 2013, 83(6):1029-1041.

31. Kim EN, Lim JH, Kim MY, Ban TH, Jang IA, Yoon HE, Park CW, Chang YS, Choi BS: Resveratrol, an Nrf2 activator, ameliorates aging-related progressive renal injury. Aging 2018, 10(1):83-99.

32. Sun YB, Ou X, Caruana G, Li J:The origin of renal fibroblasts/myofibroblasts and the signals that trigger fibrosis. Differentiation; research in biological diversity 2016, 92(3):102-107.

33. Chung AC, Huang XR, Zhou L, Heuchel R, Lai KN, Lan HY: Disruption of the Smad7 gene promotes renal fibrosis and inflammation in unilateral ureteral obstruction (UUO) in mice. Nephrology, dialysis, transplantation: official publication of the European Dialysis and Transplant Association European Renal Association 2009, 24(5):1443-1454.

34. Border WA, Noble NA: TGF-beta in kidney fibrosis: a target for gene therapy. Kidney international 1997, 51(5):1388-1396.

35. Yin Q, Liu H: Connective Tissue Growth Factor and Renal Fibrosis. Advances in experimental medicine and biology 2019, 1165:365-380.

36. Su H, Wan C, Song A, Qiu Y, Xiong W, Zhang C: Oxidative Stress and Renal Fibrosis: Mechanisms and Therapies. Advances in experimental medicine and biology 2019, 1165:585-604.

37. Zuo Y, Liu Y: New insights into the role and mechanism of Wnt/betacatenin signalling in kidney fibrosis. Nephrology 2018, 23 Suppl 4:38-43.

38. Nusse R, Clevers H:Wnt/beta-Catenin Signaling, Disease, and Emerging Therapeutic Modalities. Cell 2017, 169(6):985-999.

39. Cisternas $P$, Vio CP, Inestrosa NC: Role of Wnt signaling in tissue fibrosis, lessons from skeletal muscle and kidney. Current molecular medicine 2014, 14(4):510-522.

40. Lin X, Zha Y, Zeng XZ, Dong R, Wang QH, Wang DT: Role of the Wnt/ beta-catenin signaling pathway in inducing apoptosis and renal fibrosis in 5/6-nephrectomized rats. Molecular medicine reports 2017 15(6):3575-3582.

41. Zhou D, Tan RJ, Fu H, Liu Y: Wnt/beta-catenin signaling in kidney injury and repair: a double-edged sword. Laboratory investigation; a journal of technical methods and pathology 2016, 96(2):156-167.

42. Zhou L, Liu Y: Wnt/beta-catenin signalling and podocyte dysfunction in proteinuric kidney disease. Nature reviews Nephrology 2015 11(9):535-545.

43. Wang Y, Zhou CJ, Liu Y: Wnt Signaling in Kidney Development and Disease. Progress in molecular biology and translational science 2018, 153:181-207.

44. Zhou L, Liu Y: Wnt/beta-catenin signaling and renin-angiotensin system in chronic kidney disease. Current opinion in nephrology and hypertension 2016, 25(2):100-106.

45. Zhou G, Li J, Zeng T, Yang P, Li A: The regulatory effect of WNT-RAS signaling in hypothalamic paraventricular nucleus on renal fibrosis. J Nephrol. 2020;33(2):289-297.

46. Hall-Glenn F, Lyons KM: Roles for CCN2 in normal physiological processes. Cellular and molecular life sciences: CMLS 2011, 68(19):3209-3217.

47. Rooney B, O'Donovan H, Gaffney A, Browne M, Faherty N, Curran SP, Sadlier D, Godson C, Brazil DP, Crean J: CTGF/CCN2 activates canonical Wnt signalling in mesangial cells through LRP6: implications for the pathogenesis of diabetic nephropathy. FEBS letters 2011, 585(3):531-538.

48. Kong W: Nrf2 inhibits tubularinterstitial disease progression via regulating Wnt pathway dependent on downregulating CTGF secreation in epithelial cell doctor. China Medical University; 2018.

49. Liu B, Tu Y, He W, Liu Y, Wu W, Fang Q, Tang H, Tang R, Wan Z, Sun W et al: Hyperoside attenuates renal aging and injury induced by D-galactose via inhibiting AMPK-ULK1 signaling-mediated autophagy. Aging 2018, 10(12):4197-4212.

50. Chen W, Lin HR, Wei CM, Luo XH, Sun ML, Yang ZZ, Chen XY, Wang HB: Echinacoside, a phenylethanoid glycoside from Cistanche deserticola, extends lifespan of Caenorhabditis elegans and protects from Abetainduced toxicity. Biogerontology 2018, 19(1):47-65.

51. Xuan GD, Liu CQ: [Research on the effect of phenylethanoid glycosides (PEG) of the Cistanche deserticola on anti-aging in aged mice induced by D-galactose]. Zhong yao cai $=$ Zhongyaocai $=$ Journal of Chinese medicinal materials 2008, 31(9):1385-1388.

52. Peng XM, Gao L, Huo SX, Liu XM, Yan M: The Mechanism of Memory Enhancement of Acteoside (Verbascoside) in the Senescent Mouse 
Model Induced by a Combination of D-gal and AICl3. Phytother Res 2015, 29(8):1137-1144.

53. Tian S, Miao MS, Li XM, Bai M, Wu YY, Wei ZZ: Study on neuroendocrineimmune function of Phenylethanoid Glycosides of Desertliving Cistanche herb in perimenopausal rat model. J Ethnopharmacol 2019, 238:111884.

54. Ji SL, Cao KK, Zhao XX, Kang NX, Zhang Y, Xu QM, Yang SL, Liu YL, Wang C: Antioxidant activity of phenylethanoid glycosides on glutamate-induced neurotoxicity. Biosci Biotechnol Biochem 2019, 83(11):2016-2026.

55. Cao B, Zeng M, Si Y, Zhang B, Wang Y, Xu R, Zheng X, Feng W: Extract of Corallodiscus Flabellata Attenuates Renal Fibrosis in Aging Mice via the Wnt/B-catenin/RAS Signaling Pathway. In.: Research Square; 2020

\section{Publisher's Note}

Springer Nature remains neutral with regard to jurisdictional claims in published maps and institutional affiliations.

- fast, convenient online submission

- thorough peer review by experienced researchers in your field

- rapid publication on acceptance

- support for research data, including large and complex data types

- gold Open Access which fosters wider collaboration and increased citations

- maximum visibility for your research: over 100M website views per year

At BMC, research is always in progress.

Learn more biomedcentral.com/submissions 